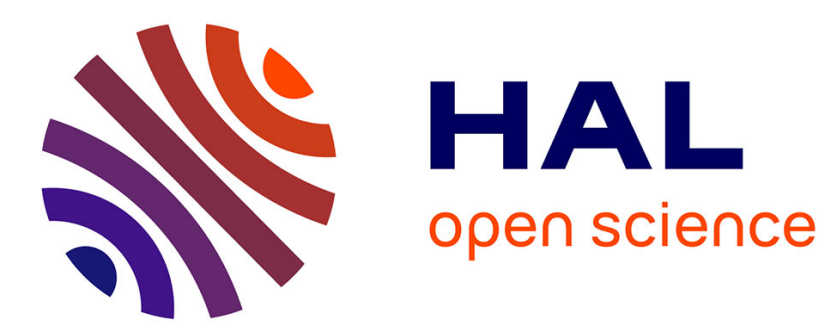

\title{
Spatial Modulation with Partial-CSI at the Receiver: Optimal Detector and Performance Evaluation
}

\author{
Marco Di Renzo, Harald Haas
}

\section{To cite this version:}

Marco Di Renzo, Harald Haas. Spatial Modulation with Partial-CSI at the Receiver: Optimal Detector and Performance Evaluation. IEEE Sarnoff Symposium, Apr 2010, United States. pp. 1-6. hal00547043

\section{HAL Id: hal-00547043 \\ https://hal.science/hal-00547043}

Submitted on 15 Dec 2010

HAL is a multi-disciplinary open access archive for the deposit and dissemination of scientific research documents, whether they are published or not. The documents may come from teaching and research institutions in France or abroad, or from public or private research centers.
L'archive ouverte pluridisciplinaire HAL, est destinée au dépôt et à la diffusion de documents scientifiques de niveau recherche, publiés ou non, émanant des établissements d'enseignement et de recherche français ou étrangers, des laboratoires publics ou privés. 


\section{Spatial Modulation with Partial-CSI at the Receiver: Optimal Detector and Performance Evaluation}

\author{
Marco Di Renzo \\ French National Center for Scientific Research (CNRS) \\ Laboratory of Signals and Systems (LSS) \\ École Supérieure d'Électricité (SUPÉLEC) \\ 3 rue Joliot-Curie, 91192 Gif-sur-Yvette (Paris), France \\ E-Mail: marco.direnzo@1ss.supelec.fr
}

\author{
Harald Haas \\ The University of Edinburgh \\ Institute for Digital Communications (IDCOM) \\ College of Science and Engineering \\ Mayfield Road, Edinburgh, EH9 3JL, UK \\ E-Mail: h.haas@ed.ac.uk
}

\begin{abstract}
Spatial Modulation (SM) is a novel and recently proposed transmission technology for Multiple-Input-MultipleOutput (MIMO) wireless communication systems, which has been shown to be a promising alternative to several popular MIMO schemes. So far, only optimal or heuristic transceivers with Full Channel State Information (F-CSI) at the receiver have been investigated, and their performance analyzed over fading channels. However, in several circumstances, channel fading might be sufficiently rapid to preclude the availability of the perfect knowledge of CSI at the receiver, and, in particular, the estimation of a stable phase reference. Motivated by this consideration, in this paper we develop the optimal detector for SM with unknown phase reference at the receiver (i.e., PartialCSI, P-CSI, knowledge), which inevitably leads to a sub-optimal receiver design. An analytical framework will be developed for the accurate performance analysis of this novel detector over fading channels, and its performance will be compared to the optimal receiver design with F-CSI. Numerical results will point out that, unlike ordinary modulation schemes, there is a substantial performance loss when the receiver cannot exploit the phase information for optimal detection operations. This result highlights the importance of accurate and reliable channel estimation mechanisms for the efficient operation of SM over fading channels. Analytical frameworks and theoretical findings will also be substantiated via Monte Carlo simulations.
\end{abstract}

\section{INTRODUCTION}

Space Shift Keying (SSK) and Spatial Modulation (SM) are two novel and recently proposed wireless transmission techniques for Multiple-Input-Multiple-Output (MIMO) wireless systems [1], [2]. Recent research efforts have pointed out that they can be promising candidates to the design of lowcomplexity modulation schemes and transceiver architectures for MIMO systems over fading channels [3]-[5]. Moreover, SSK and SM can offer better performance, with a significant reduction in receiver complexity and system design, than other MIMO communication systems, e.g., V-BLAST (Vertical Bell Laboratories Layered Space-Time), Alamouti, as well as Amplitude Phase Modulation (APM) schemes [3]-[5]. In particular, SSK is a particular instance of SM, which can reduce further the receiver complexity owing to the absence of conventional modulation schemes for data transmission [5].

The underlying and fundamental principle of SSK and SM is twofold: i) at the transmitter, a one-to-one mapping of information bits to transmit-antennas, thus allowing the transmitantenna index to convey information, and ii) at the receiver, the exploitation, due to the properties of wireless fading channels [6], of distinct multipath profiles along any transmit-receive wireless link. Moving from these basic working principles, the following contributions are available in the literature as far as optimal and sub-optimal receiver design is concerned. i) In [3], a heuristic receiver design for SM is proposed and its performance analyzed over correlated fading channels. ii) In [4], the optimal detector for SM with Full Channel State Information (F-CSI) at the receiver is developed and its performance studied over uncorrelated Rayleigh fading channels. iii) In [5], a detector for SSK, similar to [4], is introduced and its performance analyzed for uncoded and coded systems. Moreover, the authors study the effect of imperfect channel estimates when the receiver is still designed to have F-CSI. However, the results are obtained by using Monte Carlo simulations and by assuming that the estimation errors contribute to additive noise only. iv) Moving from [1], in [7] the authors propose an optimization framework to allow more than one transmit-antenna at a time to convey information and show some performance improvements due to an optimal constellation design. The optimal detector with F-CSI is always adopted.

Although the design and analysis of optimal receiver architectures are of paramount importance to get fundamental insights about receiver operations, as well as lower bounds on the achievable performance of them, there are several circumstances in which the channel fading might be sufficiently rapid to disallow the perfect knowledge of CSI at the receiver, thus precluding the possibility for a F-CSI assisted optimal receiver design [8, Ch. 14]. This consideration is theoretically and practically relevant especially for SM where, unlike conventional modulation schemes where the information is carried by a modulated signal, data information is embedded into the location-specific characteristics of the wireless channel [1], [6]. In particular, it can be easily predicted that, in a system adopting SM, neglecting part of that locationspecific channel fingerprint along any transmit-receive path may introduce some performance losses. Moving from these considerations, the main aim of the present paper is to analyze qualitatively the performance loss that is obtained when a SM detector cannot exploit channel phase information for optimal decision-making operations.

Motivated by all above considerations, in this contribution, we develop the optimal detector for SM with unknown phase reference at the receiver, thus yielding a Partial-CSI ( $\mathrm{P}-$ CSI) assisted receiver design, as opposite to F-CSI optimal detectors available in the literature so far. In particular, and without loss of generality, we will focus our attention on a $2 \times$ 1 MISO (Multiple-Input-Single-Output) SSK-based system, which represents the basic building block for understanding and designing general SM-MIMO wireless systems. While analysis and findings can be readily extended to more general 


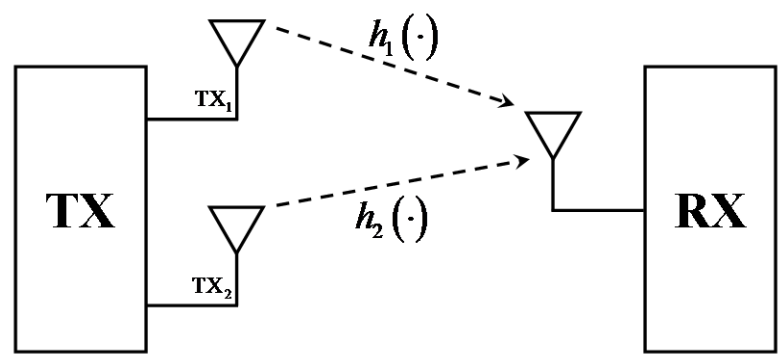

Fig. 1. System model: the $2 \times 1$ MISO setting.

system settings [9], the basic $2 \times 1$ MISO setup will allow us to concentrate the attention on the peculiar aspects of SM, and, at the same time, to keep the analytical derivation at a moderate level for a conference paper. More specifically, the main contributions of the present paper are as follows: i) the optimal receiver with unknown phase reference is derived, ii) a sub-optimal, and asymptotically tight (for high Signal-toNoise-Ratios, SNRs), version of it is proposed, and iii) an asymptotically tight approximation for performance prediction over uncorrelated Rayleigh fading channels is computed, and its accuracy validated for various system settings. Our theoretical and numerical analysis will highlight the following important outcomes. i) The optimal receiver design with PCSI results in a substantial performance loss with respect to optimal detectors with F-CSI. This result is in net contrast to ordinary modulation schemes in which the performance loss of a receiver with and without phase information is limited to a few dBs. For example, BPSK (Binary Phase Shift Keying) and DBPSK (Differential BPSK) receivers differ less than $1 \mathrm{~dB}$ over Additive White Gaussian Noise (AWGN) channels [8, Fig. 5.2.12], and approximately $3 \mathrm{~dB}$ over Rayleigh fading channels [8, Fig. 14.3.1]. ii) The performance of both F-CSI and P-CSI assisted detectors depends strongly on channel fading statistics, and, in particular, on the power imbalance between the transmit-receive paths. iii) Accordingly, accurate and reliable channel estimation mechanisms for the efficient operation of SM over fading channels appear to be of paramount importance for the deployment of SM in realistic operating environments.

The remainder of the paper is organized as follows. In Section II, system and channel models are introduced. In Section III, the optimal detector with $\mathrm{P}-\mathrm{CSI}$ is proposed along with a low-complexity implementation of it for high SNRs. In Section IV, a tight approximation for performance analysis over Rayleigh fading channels is developed. In Section V, numerical and simulation results are shown to substantiate the tightness of the proposed framework, and to compare F-CSI and P-CSI assisted detectors. Finally, Section VI concludes the paper.

\section{System Model AND BACKGROUND}

\section{A. Background}

Let us consider the $2 \times 1$ MISO system depicted in Fig. 1 . As mentioned in Section I, SSK-based transmission techniques foresee i) at the transmitted-side, to map information data bits to transmit-antenna indexes, and ii) at the receiverside, to de-map these bits via suitable detection mechanisms for estimating, for each signaling time-interval, the active transmit-antenna. In particular, the detection process at the receiver-side can be cast in terms of a general binary detection problem in AWGN [10, Sec. 4.2, pp. 254], when conditioning upon fading channel statistics. In this section, we will briefly summarize the SSK detection problem and the MaximumLikelihood (ML) optimum detector when the receiver has FCSI [4].

\section{B. Notation}

Let us briefly introduce the main notation used in what follows. i) We adopt a complex-envelope signal representation. ii) $j=\sqrt{-1}$ is the imaginary unit. iii) $(x \otimes y)(t)=$ $\int_{-\infty}^{+\infty} x(\xi) y(t-\xi) d \xi$ is the convolution of signals $x(\cdot)$ and $y(\cdot)$. iv) $(\cdot)^{*}$ denotes complex-conjugate. v) $|\cdot|^{2},|\cdot|$, and $\angle$. denote square absolute value, absolute value and phase angle of a complex vector, respectively. vi) $\mathrm{E}\{\cdot\}$ is the expectation operator vii) $\operatorname{Re}\{\cdot\}$ denotes the real part operator. viii) $\operatorname{Pr}\{\cdot\}$ means probability. ix) $Q(\cdot, \cdot)$ is the Marcum Q-function [11, Eq. (3)]. x) $\bar{m}_{1}$ and $\bar{m}_{2}$ denote the two information messages that the transmitter in Fig. 1 can emit with equal probability. xi) $\hat{m}$ denotes the message estimated at the receiver-side. xii) $E_{m}=E_{\bar{m}_{1}}=E_{\bar{m}_{2}}$ is the energy transmitted by each antenna that emits a non-zero signal. xiii) $T_{m}=T_{\bar{m}_{1}}=T_{\bar{m}_{2}}$ denotes the signaling interval for both information messages $\bar{m}_{1}$ and $\bar{m}_{2}$. xiv) The noise at the receiver input is denoted by $n(\cdot)$, and is assumed to be AWG-distributed, with both real and imaginary parts having a double-sided power spectral density equal to $\left.N_{0} . \mathrm{xv}\right)\left\{s_{i}\left(\cdot \mid\left\{\bar{m}_{n}\right\}_{n=1}^{2}\right)\right\}_{i=1}^{2}$ denote the signals emitted by the transmit-antennas $\left\{\mathrm{TX}_{i}\right\}_{i=1}^{2}$ conditioned upon the transmitted messages $\left\{\bar{m}_{i}\right\}_{i=1}^{2}$. Moreover, $w(\cdot)$ denotes the unit-energy (i.e., $\int_{-\infty}^{+\infty}|w(t)|^{2} d t=1$ ) elementary pulse waveform for each transmission. xvi) $\delta(\cdot)$ is the Dirac's delta function. xvii) $I_{\nu}(\cdot)$ is the modified Bessel function of first kind and order $\nu$ [12, Ch. 9)]. xviii) erfc (.) is the complementary error function [12, Eq. (7.1.2))]. xix) $G_{p, q}^{m, n}\left(. \mid \begin{array}{c}\left(a_{p}\right) \\ \left(b_{q}\right)\end{array}\right)$ is the Meijer-G function defined in [13, Ch. 8, pp. 519]. XX) $\left\{\psi_{p}\right\}_{p=1}^{N_{p}}$ and $\left\{\zeta_{p}\right\}_{p=1}^{N_{p}}$ are weights and abscissas of the $N_{p^{-}}$ order Gauss-Legendre Quadrature Rule (GLQR) [12, Table 25.10)].

\section{Channel Model}

We consider a frequency-flat slowly-varying fading channel model, with fading envelopes distributed according to a Rayleigh distribution [14, Sec. 2.2.1.1]. Moreover, we assume independent but not necessarily identically distributed fading envelopes. In particular:

- $\left\{h_{i}(t)\right\}_{i=1}^{2}=\beta_{i} \exp \left(j \varphi_{i}\right) \delta\left(t-\tau_{i}\right)$ is the channel impulse response from the $i$-th transmit-antenna to the single receive antenna of the MISO system depicted in Fig. 1, with $\left\{\beta_{i}\right\}_{i=1}^{2},\left\{\varphi_{i}\right\}_{i=1}^{2}$, and $\left\{\tau_{i}\right\}_{i=1}^{2}$ denoting gain, phase, and delay of the related wireless link. Moreover, $\left\{\alpha_{i}\right\}_{i=1}^{2}=\beta_{i} \exp \left(j \varphi_{i}\right)$ denotes the channel complexgain of $i$-th transmit-receive path.

- According to a Rayleigh fading channel model, the channel phases $\left\{\varphi_{i}\right\}_{i=1}^{2}$ are independent and uniformly distributed Random Variables (RVs) in $[0,2 \pi)$, and the fading envelopes $\left\{\beta_{i}\right\}_{i=1}^{2}$ have the following Probability 
Density Function (PDF) [14, Eq. (2.6)]:

$$
\left\{f_{\beta_{i}}(\xi)\right\}_{i=1}^{2}=\frac{2 \xi}{\Omega_{i}} \exp \left(-\frac{\xi^{2}}{\Omega_{i}}\right)
$$

where $\left\{\Omega_{i}\right\}_{i=1}^{2}=\mathrm{E}\left\{\beta_{i}^{2}\right\}$.

- $\left\{\tau_{i}\right\}_{i=1}^{M}$ are assumed to be independent and uniformly distributed in $\left[0, T_{m}\right)$, but perfectly known at the receiver, i.e., perfect time-synchronization is considered.

\section{Optimal Detector with F-CSI}

Moving from the above system and channel model, the signals after propagation through the wireless fading channel for both wireless links are $\left\{\tilde{s}_{i}\left(t \mid\left\{\bar{m}_{n}\right\}_{n=1}^{2}\right)\right\}_{i=1}^{2}=$ $\left(s_{i} \otimes h_{i}\right)(t)=\beta_{i} \exp \left(j \varphi_{i}\right) s_{i}\left(t-\tau_{i} \mid \bar{m}_{n}\right)$, and the received signal can be written as follows:

$$
\left\{\begin{array}{l}
r\left(t \mid \bar{m}_{1}\right)=\tilde{s}_{1}\left(t \mid \bar{m}_{1}\right)+\tilde{s}_{2}\left(t \mid \bar{m}_{1}\right)+n(t) \\
r\left(t \mid \bar{m}_{2}\right)=\tilde{s}_{1}\left(t \mid \bar{m}_{2}\right)+\tilde{s}_{2}\left(t \mid \bar{m}_{2}\right)+n(t)
\end{array}\right.
$$

when messages $\bar{m}_{1}$ and $\bar{m}_{2}$ are transmitted, respectively. Note that (2) is a general hypothesis binary testing problem where both transmit-antennas could be activated when a message is transmitted [1], [7].

Accordingly, for SSK modulation the following general binary detection problem in (3) can be formulated:

$$
\begin{cases}r(t)=\bar{s}_{1}(t)+n(t) & \text { if } \bar{m}_{1} \text { is sent } \\ r(t)=\bar{s}_{2}(t)+n(t) & \text { if } \bar{m}_{2} \text { is sent }\end{cases}
$$

where $\bar{s}_{1}(t)=\tilde{s}_{1}\left(t \mid \bar{m}_{1}\right)+\tilde{s}_{2}\left(t \mid \bar{m}_{1}\right)$ and $\bar{s}_{2}(t)=$ $\tilde{s}_{1}\left(t \mid \bar{m}_{2}\right)+\tilde{s}_{2}\left(t \mid \bar{m}_{2}\right)$.

Moving from (3), the ML optimum detector with F-CSI and perfect time-synchronization at the receiver is as follows [10, Sec. 4.2, pp. 254, eq. (31)]:

$$
\hat{m}= \begin{cases}\bar{m}_{1} & \text { if } D_{1} \geq D_{2} \\ \bar{m}_{2} & \text { if } D_{2}<D_{1}\end{cases}
$$

where $\left\{D_{i}\right\}_{i=1}^{2}$ are the decision metrics defined as follows:

$\left\{\begin{array}{l}D_{1}=\exp \left[\frac{1}{N_{0}} \operatorname{Re}\left\{\int_{T_{m}} r(t) \bar{s}_{1}^{*}(t) d t\right\}-\frac{1}{2 N_{0}} \int_{T_{m}} \bar{s}_{1}(t) \bar{s}_{1}^{*}(t) d t\right] \\ D_{2}=\exp \left[\frac{1}{N_{0}} \operatorname{Re}\left\{\int_{T_{m}} r(t) \bar{s}_{2}^{*}(t) d t\right\}-\frac{1}{2 N_{0}} \int_{T_{m}} \bar{s}_{2}(t) \bar{s}_{2}^{*}(t) d t\right]\end{array}\right.$

According to [3]-[5], for SSK modulation we have:

$$
\left\{\begin{array}{l}
\bar{s}_{1}(t)=\tilde{s}_{1}\left(t \mid \bar{m}_{1}\right) \\
\bar{s}_{2}(t)=\tilde{s}_{2}\left(t \mid \bar{m}_{2}\right)
\end{array}\right.
$$

which means that only one transmit-antenna is activated when either $\bar{m}_{1}$ or $\bar{m}_{2}$ are sent, i.e., $s_{1}\left(t \mid \bar{m}_{2}\right)=s_{2}\left(t \mid \bar{m}_{1}\right)=0$.

By letting $s_{1}\left(t \mid \bar{m}_{1}\right)=s_{2}\left(t \mid \bar{m}_{2}\right)=\sqrt{E_{m}} w(t)$, (2) simplifies as follows $\left(t \in\left[\bar{\tau}, \bar{\tau}+T_{m}\right)\right)$ :

$$
\left\{\begin{array}{l}
r\left(t \mid \bar{m}_{1}\right)=\sqrt{E_{m}} \beta_{1} \exp \left(j \varphi_{1}\right) w(t)+n(t) \\
r\left(t \mid \bar{m}_{2}\right)=\sqrt{E_{m}} \beta_{2} \exp \left(j \varphi_{2}\right) w(t)+n(t)
\end{array}\right.
$$

which implicitly assumes that either i) a pure sinusoidal tone is transmitted, i.e., $w(t)=1$, and the propagation delays are embedded, with a slight abuse of notation, into the channel phases, or ii) $\tau_{1} \cong \tau_{2}=\bar{\tau}$, which is a realistic assumption when the distance between transmitter and receiver is much larger than the spacing between the transmit-antennas.

\section{Optimal Detector With P-CSI}

The optimal detector in (5) implicitly assumes the a priori knowledge at the receiver of both fading envelopes $\left\{\beta_{i}\right\}_{i=1}^{2}$ and phases $\left\{\varphi_{i}\right\}_{i=1}^{2}$. In this section, we will compute the optimal detector with unknown phase information. In particular, this latter detector can be obtained by substituting (6) into (5), which results in the following decision metrics:

$$
\left\{D_{i}\left(\varphi_{i}\right)\right\}_{i=1}^{2}=\exp \left[\frac{\sqrt{E_{m}} \beta_{i}}{N_{0}}|\bar{r}| \cos \left(\varphi_{i}-\angle \bar{r}\right)-\frac{E_{m} \beta_{i}^{2}}{2 N_{0}}\right]_{(8)}
$$

where we have explicitly emphasized that $D_{1}$ and $D_{2}$ are now conditioned upon the channel phases $\left\{\varphi_{i}\right\}_{i=1}^{2}$, i.e., $\left\{D_{i}\right\}_{i=1}^{2}=$ $D_{i}\left(\varphi_{i}\right)$, as well as defined $\bar{r}=\int_{T_{m}} r(t) w^{*}(t) d t=$ $|\bar{r}| \exp (j \angle \bar{r})$.

By averaging $\left\{D_{i}(\cdot)\right\}_{i=1}^{2}$ in (8) over the distribution of $\left\{\varphi_{i}\right\}_{i=1}^{2}$ using analytical steps similar to [10, pp. 339, Eq. (366), Eq. (367)], and then computing the logarithm of the obtained result, i.e., $\left\{\bar{D}_{i}\right\}_{i=1}^{2}=\ln \left[(1 / 2 \pi) \int_{0}^{2 \pi} D_{i}\left(\phi_{i}\right) d \phi_{i}\right]$, we obtain:

$$
\left\{\bar{D}_{i}\right\}_{i=1}^{2}=\ln \left[I_{0}\left(\frac{\sqrt{E_{m}} \beta_{i}}{N_{0}}|\bar{r}|\right)\right]-\frac{E_{m} \beta_{i}^{2}}{2 N_{0}}
$$

which yields the optimal detector with P-CSI at the receiver and agrees, e.g., with [14, Eq. (7.24)].

The analysis of the detector in (9) is quite cumbersome due to the Bessel function $I_{0}(\cdot)$ that needs to be computed. A simpler and asymptotically (for high SNRs) equivalent detector can be obtained by simply recognizing that $\ln \left[I_{0}(|x|)\right] \cong|x|$ when $|x| \gg 1$. By exploiting this asymptotic approximation, the detector in (9) simplifies as follows:

$$
\left\{\bar{D}_{i}\right\}_{i=1}^{2}=\sqrt{E_{m}} \beta_{i}|\bar{r}|-\frac{E_{m} \beta_{i}^{2}}{2}
$$

where irrelevant constants have also been neglected.

By carefully looking at (10), we can observe that $\left\{\bar{D}_{i}\right\}_{i=1}^{2}$ is very similar to the heuristic detector used in [3, Eq. (3) $]$ for estimating the antenna index at the receiver. They differ for the bias factor $E_{m} \beta_{i}^{2} / 2$, which allows to cope with the assumption of constrained channels remarked in [4]. In other words, the detector in [3, Eq. (3)] is not completely heuristic, but can be interpreted, apart from the bias term discussed above, as a high SNR approximation of the optimal detector with unknown phase reference at the receiver. This is an understandable outcome when it is observed that the detector in [3, Eq. (3)] is used to make the detection process of the antenna index independent of the modulated data transmitted by each antenna.

\section{Average Bit Error Probability (ABEP)}

In this section, we will develop an accurate analytical framework for performance analysis of the detector in (10). We will soon realize that, while to develop the detector in (9) and (10) well-known techniques have been used, the computation of the ABEP over fading channels will require novel analytical approaches to deal with the specific signal structure of SSK modulation. To efficiently handle the complexity of this problem, a tight approximation will be proposed.

\section{A. Conditional BEP: Fixed Channel Realization}

From the decision rule in (4), the probability of error, $\mathrm{P}_{\mathrm{E}}$, of the detection process (i.e., the detection of the index of 


$$
\begin{gathered}
\mathrm{P}_{\mathrm{E}}\left(h_{1}, h_{2}\right)=\left[\frac{1}{2}-\frac{1}{2} Q\left(\frac{\sqrt{E_{m}} \beta_{1}}{\sqrt{N_{0}}}, \frac{\sqrt{E_{m}}\left(\beta_{1}+\beta_{2}\right)}{2 \sqrt{N_{0}}}\right)+\frac{1}{2} Q\left(\frac{\sqrt{E_{m}} \beta_{2}}{\sqrt{N_{0}}}, \frac{\sqrt{E_{m}}\left(\beta_{1}+\beta_{2}\right)}{2 \sqrt{N_{0}}}\right)\right] \cdot \operatorname{Pr}\left\{\beta_{1} \geq \beta_{2}\right\} \\
+\left[\frac{1}{2}-\frac{1}{2} Q\left(\frac{\sqrt{E_{m}} \beta_{2}}{\sqrt{N_{0}}}, \frac{\sqrt{E_{m}}\left(\beta_{1}+\beta_{2}\right)}{2 \sqrt{N_{0}}}\right)+\frac{1}{2} Q\left(\frac{\sqrt{E_{m}} \beta_{1}}{\sqrt{N_{0}}}, \frac{\sqrt{E_{m}}\left(\beta_{1}+\beta_{2}\right)}{2 \sqrt{N_{0}}}\right)\right] \cdot \operatorname{Pr}\left\{\beta_{1}<\beta_{2}\right\} \\
Q(a, b) \leq \frac{I_{0}(a b)}{\exp (a b)}\left\{\exp \left[-\frac{(b-a)^{2}}{2}\right]+a \sqrt{\frac{\pi}{2}} \operatorname{erfc}\left(\frac{b-a}{\sqrt{2}}\right)\right\} \cong \underbrace{\frac{1}{\sqrt{2 \pi a b}} \exp \left[-\frac{(b-a)^{2}}{2}\right]}_{P_{1}(a, b)}+\underbrace{\frac{1}{2} \sqrt{\frac{a}{b}} \operatorname{erfc}\left(\frac{b-a}{\sqrt{2}}\right)}_{P_{2}(a, b)}
\end{gathered}
$$

$$
\begin{aligned}
1-Q(a, b) & \geq \frac{I_{0}(a b)}{\exp (a b)}\left\{\exp \left(-\frac{a^{2}}{2}\right)-\exp \left[-\frac{(b-a)^{2}}{2}\right]+a \sqrt{\frac{\pi}{2}} \operatorname{erfc}\left(-\frac{a}{\sqrt{2}}\right)-a \sqrt{\frac{\pi}{2}} \operatorname{erfc}\left(\frac{b-a}{\sqrt{2}}\right)\right\} \\
& \cong \underbrace{\frac{1}{\sqrt{2 \pi a b}} \exp \left(-\frac{a^{2}}{2}\right)}_{P_{3}(a, b)}-\underbrace{\frac{1}{\sqrt{2 \pi a b}} \exp \left[-\frac{(b-a)^{2}}{2}\right]}_{P_{1}(a, b)}+\underbrace{\frac{1}{2} \sqrt{\frac{a}{b} \operatorname{erfc}\left(-\frac{a}{\sqrt{2}}\right)}}_{P_{4}(a, b)}-\underbrace{\frac{1}{2} \sqrt{\frac{a}{b}} \operatorname{erfc}\left(\frac{b-a}{\sqrt{2}}\right)}_{P_{2}(a, b)}
\end{aligned}
$$

$$
\begin{aligned}
\mathrm{P}_{\mathrm{E}}\left(h_{1}, h_{2}\right) \cong & \frac{1}{2} \sum_{k=1}^{4}\left[\vartheta_{k} P_{k}\left(\gamma_{1}, \gamma_{1,2}\right) \operatorname{Pr}\left\{\beta_{1} \geq \beta_{2}\right\}\right]+\frac{1}{2} \sum_{k=1}^{2}\left[P_{k}\left(\gamma_{2}, \gamma_{1,2}\right) \operatorname{Pr}\left\{\beta_{1} \geq \beta_{2}\right\}\right] \\
& +\frac{1}{2} \sum_{k=1}^{4}\left[\vartheta_{k} P_{k}\left(\gamma_{2}, \gamma_{1,2}\right) \operatorname{Pr}\left\{\beta_{1}<\beta_{2}\right\}\right]+\frac{1}{2} \sum_{k=1}^{2}\left[P_{k}\left(\gamma_{1}, \gamma_{1,2}\right) \operatorname{Pr}\left\{\beta_{1}<\beta_{2}\right\}\right]
\end{aligned}
$$

the transmit-antenna), when conditioning upon the channel impulses responses $\left\{h_{i}(\cdot)\right\}_{i=1}^{2}$, is as follows:

$$
\begin{aligned}
& \mathrm{P}_{\mathrm{E}}\left(h_{1}, h_{2}\right)=\left.\frac{1}{2} \mathrm{P}_{\mathrm{E}}\left(h_{1}, h_{2}\right)\right|_{\bar{m}_{1}}+\left.\frac{1}{2} \mathrm{P}_{\mathrm{E}}\left(h_{1}, h_{2}\right)\right|_{\bar{m}_{2}} \\
& =\frac{1}{2} \operatorname{Pr}\left\{\left.D_{1}\right|_{\bar{m}_{1}}<\left.D_{2}\right|_{\bar{m}_{1}}\right\}+\frac{1}{2} \operatorname{Pr}\left\{\left.D_{2}\right|_{\bar{m}_{2}}<\left.D_{1}\right|_{\bar{m}_{2}}\right\}
\end{aligned}
$$

where $\left\{\left.\mathrm{P}_{\mathrm{E}}(\cdot, \cdot)\right|_{\bar{m}_{i}}\right\}_{i=1}^{2}$ and $\left\{\left.D_{l}\right|_{\bar{m}_{i}}\right\}_{i, l=1}^{2}$ denote the probabilities of error and the decision metrics conditioned upon the transmission of messages $\left\{\bar{m}_{i}\right\}_{i=1}^{2}$, respectively.

Let us compute $\left.\mathrm{P}_{\mathrm{E}}(\cdot, \cdot)\right|_{\bar{m}_{1}}$. By substituting (7) in (10), and after a few algebraic manipulations, which are here omitted due to space constraints, we obtain (12) on top of this page, where we have defined $\bar{n}=\int_{T_{m}} n(t) w^{*}(t) d t$. Moreover, we can readily recognize that $R_{1}=\left|\sqrt{E_{m}} \beta_{1} \exp \left(j \varphi_{1}\right)+\bar{n}\right|$ is a Rice-distributed RV with Cumulative Distribution Function $(\mathrm{CDF}), F_{R_{1}}(\cdot)$, equal to $F_{R_{1}}(\xi)=1-Q(s / \sigma, r / \sigma)$ [8, Eq. 2.1.142] with $s=\sqrt{E_{m}} \beta_{1}$ and $\sigma=\sqrt{N_{0}}$. Accordingly, (12) reduces as shown in what follows:

$\left.\mathrm{P}_{\mathrm{E}}\left(h_{1}, h_{2}\right)\right|_{\bar{m}_{1}}= \begin{cases}1-Q\left(\frac{\sqrt{E_{m}} \beta_{1}}{\sqrt{N_{0}}}, \frac{\sqrt{E_{m}}\left(\beta_{1}+\beta_{2}\right)}{2 \sqrt{N_{0}}}\right) & \text { if } \beta_{1} \geq \beta_{2} \\ Q\left(\frac{\sqrt{E_{m}} \beta_{1}}{\sqrt{N_{0}}}, \frac{\sqrt{E_{m}}\left(\beta_{1}+\beta_{2}\right)}{2 \sqrt{N_{0}}}\right) & \text { if } \beta_{1}<\beta_{2}\end{cases}$

With similar analytical steps, which are here omitted due to space constraints, we can obtain a similar result for $\left.\mathrm{P}_{\mathrm{E}}(\cdot, \cdot)\right|_{\bar{m}_{2}}$ :

$\left.\mathrm{P}_{\mathrm{E}}\left(h_{1}, h_{2}\right)\right|_{\bar{m}_{2}}= \begin{cases}1-Q\left(\frac{\sqrt{E_{m}} \beta_{2}}{\sqrt{N_{0}}}, \frac{\sqrt{E_{m}}\left(\beta_{1}+\beta_{2}\right)}{2 \sqrt{N_{0}}}\right) & \text { if } \beta_{2} \geq \beta_{1} \\ Q\left(\frac{\sqrt{E_{m}} \beta_{2}}{\sqrt{N_{0}}}, \frac{\sqrt{E_{m}}\left(\beta_{1}+\beta_{2}\right)}{2 \sqrt{N_{0}}}\right) & \text { if } \beta_{2}<\beta_{1}\end{cases}$

In summary, the (conditional) error probability in (11) can be computed as shown in (15) on top of this page. We will show in Section IV-C that the probabilities $\operatorname{Pr}\left\{\beta_{1} \geq \beta_{2}\right\}$ and $\operatorname{Pr}\left\{\beta_{1}<\beta_{2}\right\}$ do not need to be actually computed to obtain the ABEP.

\section{B. Tight Approximation for $\mathrm{P}_{\mathrm{E}}(\cdot, \cdot)$}

By looking at (15), it is simple to understand that removing the conditioning over the wireless channel statistics can be quite involving. As a matter of fact, in this specific case we are unable to use well consolidated tools to re-write the Marcum Q-function in (15) in an equivalent integral form useful for averaging over the distribution of the fading envelopes [15, Eq. (14a), Eq. (14b)]. To circumvent this problem, we propose to jointly use an upper and a lower bound that exploit the results in [11], which are offered in a suitable form to solve the problem at hand.

In particular, to approximate $\mathrm{P}_{\mathrm{E}}(\cdot, \cdot)$ in (15) we use the upper and lower bounds for the Marcum-Q function summarized in (16) and (17) on top of this page. These bounds are taken from [11, Eq. (7)] and [11, Eq. (12)], respectively. Furthermore, to reduce further the analytical complexity, we have exploited the approximation in [16, Eq. (8)] for the $I_{0}(\cdot)$ Bessel function. Accordingly, by substituting (16) and (17) into (15), $\mathrm{P}_{\mathrm{E}}(\cdot, \cdot)$ can be tightly approximated as shown in (18) on top of this page, where we have defined $\gamma_{1}=\beta_{1} \sqrt{E_{m} / N_{0}}$, $\gamma_{2}=\beta_{2} \sqrt{E_{m} / N_{0}}, \gamma_{1,2}=(1 / 2)\left(\beta_{1}+\beta_{2}\right) \sqrt{E_{m} / N_{0}}$, and $\left\{\vartheta_{k}\right\}_{k=1}^{4}=\{-1,-1,1,1\}$.

\section{ABEP over Rayleigh Fading}

We observe that the final result in (18) is now in a very convenient form to be averaged over fading channel statistics $\left(\beta_{1}, \beta_{2}\right)$, given that the conditional BEP is expressed as the summation of elementary functions. In particular, the ABEP can be readily written as shown in what follows:

$$
\begin{aligned}
\mathrm{ABEP} & \cong \frac{1}{2} \sum_{k=1}^{4}\left[\vartheta_{k} P_{k}^{>}(1)\right]+\frac{1}{2} \sum_{k=1}^{2}\left[P_{k}^{>}(2)\right] \\
& +\frac{1}{2} \sum_{k=1}^{4}\left[\vartheta_{k} P_{k}^{<}(2)\right]+\frac{1}{2} \sum_{k=1}^{2}\left[P_{k}^{<}(1)\right]
\end{aligned}
$$




$$
P_{2}^{>}(2)=\frac{2 \sqrt{2}}{\Omega_{1} \Omega_{2}} \int_{0}^{+\infty} \xi_{1} \exp \left(-\frac{\xi_{1}^{2}}{\Omega_{1}}\right) \underbrace{\left[\int_{0}^{\xi_{1}} \xi_{2}^{3 / 2}\left(\xi_{1}+\xi_{2}\right)^{-(1 / 2)} \operatorname{erfc}\left(\frac{\bar{\gamma}\left(\xi_{2}-\xi_{1}\right)}{2 \sqrt{2}}\right) \exp \left(-\frac{\xi_{2}^{2}}{\Omega_{2}}\right) d \xi_{2}\right]}_{J\left(\xi_{1}\right)} d \xi_{1}
$$

$$
\begin{aligned}
P_{2}^{>}(2) & =\frac{2 \sqrt{2}}{\Omega_{1} \Omega_{2}} \sum_{p=1}^{N_{p}}\left\{\frac{\psi_{p} \zeta_{p}^{3 / 2}}{\left(1+\zeta_{p}\right)^{1 / 2}} \int_{0}^{+\infty} \xi_{1}^{3} \operatorname{erfc}\left(\frac{\bar{\gamma}\left(1-\zeta_{p}\right)}{2 \sqrt{2}} \xi_{1}\right) \exp \left[-\left(\frac{1}{\Omega_{1}}+\frac{\zeta_{p}^{2}}{\Omega_{2}}\right) \xi_{1}^{2}\right] d \xi_{1}\right\} \\
& =\frac{\sqrt{2 / \pi}}{\Omega_{1} \Omega_{2}} \sum_{p=1}^{N_{p}}\left\{\frac{\psi_{p} \zeta_{p}^{3 / 2}}{\left(1+\zeta_{p}\right)^{1 / 2}}\left(\frac{1}{\Omega_{1}}+\frac{\zeta_{p}^{2}}{\Omega_{2}}\right)^{-2} G_{2,2}^{2,1}\left(\frac{\left[\frac{\bar{\gamma}\left(1-\zeta_{p}\right)}{2 \sqrt{2}}\right]^{2}}{\frac{1}{\Omega_{1}}+\frac{\zeta_{p}^{2}}{\Omega_{2}}} \mid \begin{array}{cc}
-1 & 1 \\
0 & \frac{1}{2}
\end{array}\right)\right\}
\end{aligned}
$$

where we have defined $\left\{P_{k}^{>}(i)\right\}_{i=1}^{2}$ E $\left\{P_{k}\left(\gamma_{i}, \gamma_{1,2}\right) \operatorname{Pr}\left\{\beta_{1} \geq \beta_{2}\right\}\right\}, \quad\left\{P_{k}^{<}(i)\right\}_{i=1}^{2}$ $\mathrm{E}\left\{P_{k}\left(\gamma_{i}, \gamma_{1,2}\right) \operatorname{Pr}\left\{\beta_{1}<\beta_{2}\right\}\right\}$, and ABEP $\mathrm{E}\left\{\mathrm{P}_{\mathrm{E}}\left(h_{1}, h_{2}\right)\right\}$.

Furthermore, each expectation in (19) can be computed from the definition of the expectation operator, as follows:

$$
\left\{\begin{array}{l}
\left\{P_{k}^{>}(i)\right\}_{i=1}^{2}=\int_{0}^{+\infty} \int_{0}^{\xi_{1}} P_{k}\left(\gamma_{i}, \gamma_{1,2}\right) f_{\beta_{1}}\left(\xi_{1}\right) f_{\beta_{2}}\left(\xi_{2}\right) d \xi_{1} d \xi_{2} \\
\left\{P_{k}^{<}(i)\right\}_{i=1}^{2}=\int_{0}^{+\infty} \int_{0}^{\xi_{2}} P_{k}\left(\gamma_{i}, \gamma_{1,2}\right) f_{\beta_{1}}\left(\xi_{1}\right) f_{\beta_{2}}\left(\xi_{2}\right) d \xi_{1} d \xi_{2}
\end{array}\right.
$$

where $\left\{f_{\beta_{i}}(\cdot)\right\}_{i=1}^{2}$ can be found in (1).

Due to space constraints, we are unable to provide the final result for each integral term in (20). However, the interested reader may find this derivation in [9]. For the sake of illustration, we show, in what follows, the analytical steps needed to compute one of the above integrals. The rest of the integrals can be obtained by following the same methodology.

\section{Case Study: Computation of $P_{2}^{>}(2)$}

Let us consider the computation of $P_{2}^{>}(2)$. It can be explicitly re-written as shown in (21) on top of this page, where we have defined $\bar{\gamma}=\sqrt{E_{m} / N_{0}}$. The integral $J(\cdot)$ into the square brackets in (21) can be solved by first using the change of variable $x_{2}=\xi_{2} / \xi_{1}$ and then using GLQR. The final result is shown in (22) in what follows ${ }^{1}$ :

$$
J\left(\xi_{1}\right)=\xi_{1}^{2} \sum_{p=1}^{N_{p}}\left[\frac{\psi_{p} \zeta_{p}^{3 / 2}}{\left(1+\zeta_{p}\right)^{1 / 2}} \operatorname{erfc}\left(\frac{\bar{\gamma}\left(1-\zeta_{p}\right) \xi_{1}}{2 \sqrt{2}}\right) \exp \left(-\frac{\zeta_{p}^{2}}{\Omega_{2}} \xi_{1}^{2}\right)\right]
$$

By substituting (22) into (21) and using [13, Eq. (8.4.3.1)], [13, Eq. (8.4.14.2)] along with the Mellin-Barnes integration theorem in [13, Eq. (2.24.1.1)], we get the final result in (23) on top of this page.

\section{Numerical And Simulation Results}

In this section, we show some numerical results in order to substantiate the accuracy of the proposed analytical framework for performance analysis of sub-optimal receiver designs for SSK modulation with P-CSI, as well as compare their performance with optimal receiver schemes with F-CSI.

\footnotetext{
${ }^{1}$ The interested reader may refer to [17] for issues related to truncation errors and convergence conditions of GLQR integration.
}

$=$ System setup and parameter setting can be found in the figures captions and legends.

In Figs. 2, 3, we have compared the ABEP obtained with the analytical model in (19) with both P-CSI detectors developed in (9) and (10), respectively. In particular, in Fig. 2 we can observe that although the ABEP is computed by assuming the detector in (10), which is a high SNR approximation of (9), our framework can well capture the behavior of (10) even for moderate SNRs. As expected, in the high SNR region the framework is asymptotically tight. In Fig. 3, the same analytical model is compared with the simpler detector in (10). As expected, the model is even more accurate than in Fig. 2, especially in the very low SNR region. These results lead to the following general conclusion. i) The detector in (10) yields similar performance as (9) but with a significant reduction in computational complexity since the computation of the Bessel function is avoided. ii) The proposed model is very accurate, and, although exploiting both a lower and a upper bound, it is asymptotically tight as both bounds are asymptotically accurate for high SNRs.

In Fig. 4, we have shown the ABEP of the optimal detector with F-CSI described in Section II-D. By carefully comparing it with Figs. 2, 3, we can readily conclude that neglecting the channel phase information for a low-complexity receiver implementation (i.e., keeping the channel estimator at low complexity) may lead to a substantial performance loss with respect to a F-CSI system design. This result is in net contrast to ordinary modulation schemes in which the performance loss of a receiver with and without phase information is limited to a few dBs. This performance drop is due to the peculiar operations of SM and SSK modulation with respect to ordinary modulation schemes: since it is the location-specific channel fingerprint which carries information, by neglecting part of it (e.g., the channel phase in this paper) may lead to a significant performance degradation. As a consequence of that, the design and adoption of accurate channel estimation algorithms will play a fundamental role for the successful exploitation and deployment of SM and SSK modulation concepts in realistic environments.

Finally, by carefully analyzing Figs. $2-4$ we can get important insights about the effect of channel fading on the system performance, for both F-CSI and P-CSI receiver implementations. In particular, we note that the more the transmit-receive paths are unbalanced (i.e., $\Omega_{1} \neq \Omega_{2}$ ), the better the ABEP is. Moreover, the performance improvement can be significant: up to $10 \mathrm{~dB}$ in performance gain can be 


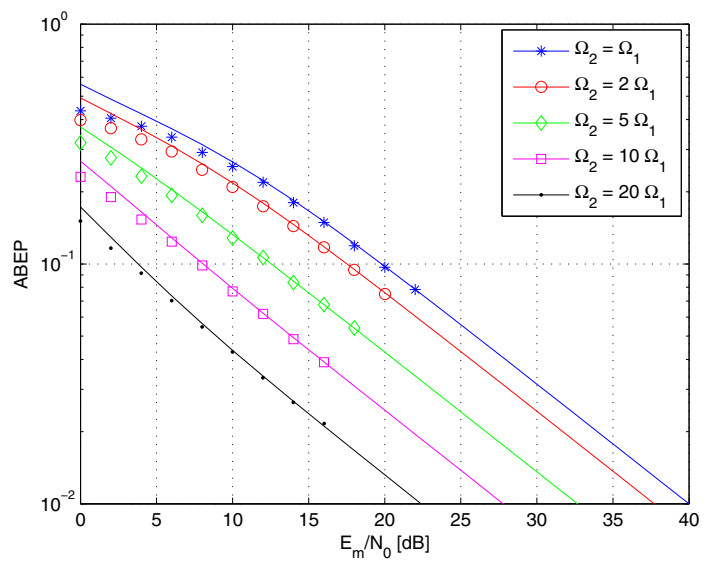

Fig. 2. Receiver with P-CSI. Comparison between the analytical model (solid lines) in (19) and Monte Carlo simulations (markers) using the Bessel-type detector in (9).

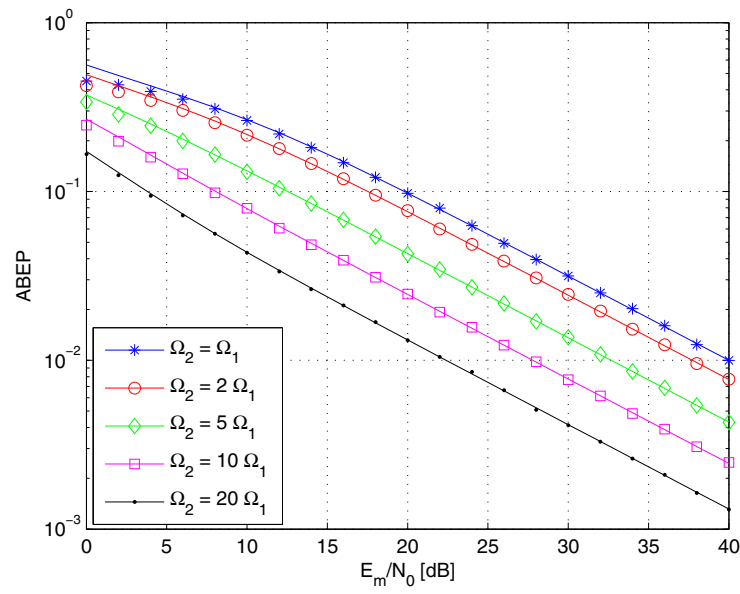

Fig. 3. Receiver with P-CSI. Comparison between the analytical model (solid lines) in (19) and Monte Carlo simulations (markers) using the simpler detector in (10).

observed moving from $\Omega_{1}=\Omega_{2}$ to $\Omega_{2}=20 \Omega_{1}$. The reason for this behavior is simple: the more the channels are different on average (i.e., $\Omega_{1} \neq \Omega_{2}$ ), the more they are distinguishable from each other, and channel fading can only occasionally lead to wrong decisions. In other words, the scenario with identically distributed fading is the worst case study for the analysis of SM and SSK modulation schemes when the channel fading can be assumed to be uncorelated. This result naturally leads to the conclusion that SM and SSK modulation schemes are inherently suitable for operation in distributed MIMO settings, where it is more likely that the transmit-receive paths are more unbalanced with respect to each other, and, so, the channel impulse responses linked to them are more distinguishable.

\section{CONCLUSION}

In this paper, we have developed and analyzed the performance of the optimal detector for SSK modulation with P-CSI at the receiver. Numerical results have confirmed the accurate performance prediction offered by the proposed analytical framework, as well as the substantial performance loss of PCSI assisted receivers when compared to F-CSI ones.

\section{ACKNOWLEDGMENT}

We gratefully acknowledge support from the EPSRC (EP/G011788/1) for this work. Harald Haas acknowledges the

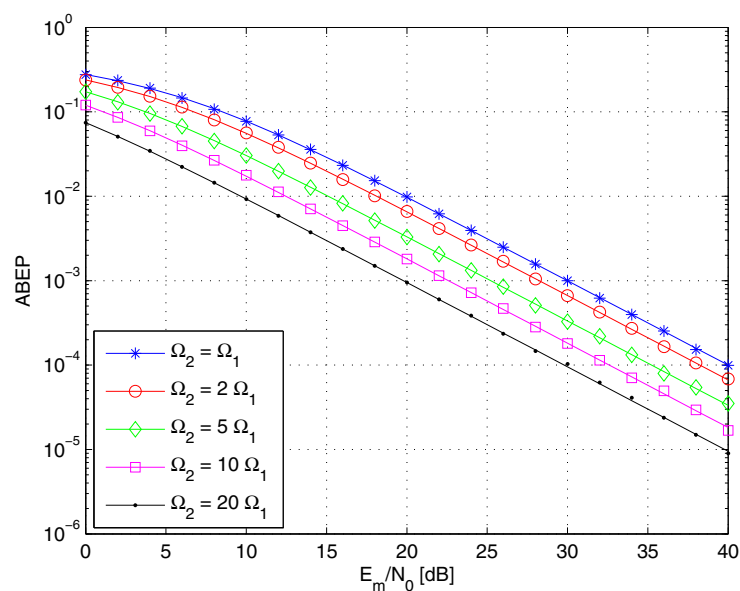

Fig. 4. Receiver with F-CSI. Comparison between the analytical model (solid lines) in [5] and Monte Carlo simulations (markers) using the detector in Section II-D.

Scottish Funding Council support of his position within the Edinburgh Research Partnership in Engineering and Mathematics between the University of Edinburgh and Heriot Watt University.

\section{REFERENCES}

[1] Y. Chau and S.-H. Yu, "Space modulation on wireless fading channels", IEEE Veh. Technol. Conf. - Fall, vol. 3, pp. 1668-1671, Oct. 2001.

[2] H. Haas, E. Costa, and E. Schultz, "Increasing spectral efficiency by data multiplexing using antennas arrays", IEEE Int. Symp. Personal, Indoor, Mobile Radio Commun., vol. 2, pp. 610-613, Sept. 2002.

[3] R. Y. Mesleh, H. Haas, S. Sinanovic, C. W. Ahn, and S. Yun, "Spatial modulation", IEEE Trans. Veh. Technol., vol. 57, no. 4, pp. 2228-2241, July 2008.

[4] J. Jeganathan, A. Ghrayeb, and L. Szczecinski, "Spatial modulation: Optimal detection and performance analysis", IEEE Commun. Lett., vol 12, no. 8, pp. 545-547, Aug. 2008.

[5] J. Jeganathan, A. Ghrayeb, L. Szczecinski, and A. Ceron, "Space shift keying modulation for MIMO channels", IEEE Trans. Wireless Commun., vol. 8, no. 7, pp. 3692-3703, July 2009.

[6] L. Xiao, L. Greenstein, N. Mandayam, and W. Trappe, "Using the physical layer for wireless authentication in time-variant channels", IEEE Trans. Wireless Commun., vol. 7, no. 7, pp. 2571-2579, July 2008.

[7] J. Jeganathan, A. Ghrayeb, and L. Szczecinski, "Generalized space shift keying modulation for MIMO channels", IEEE Int. Symp. Personal, Indoor, Mobile Radio Commun., pp. 1-5, Sept. 2008.

[8] J. Proakis, Digital Communications, McGraw-Hill, 4th ed., 2000.

[9] M. Di Renzo and H. Haas, "Space Shift Keying (SSK) modulation with partial channel state information at the receiver: Optimal detector and performance analysis over correlated fading channels", IEEE Trans. Commun., Feb. 2010 (accepted with minor revision).

[10] H. L. Van Trees, Detection, Estimation, and Modulation Theory, Part I: Detection, Estimation, and Linear Modulation Theory, John Wiley \& Sons, Inc. 2001, ISBNs: 0-471-09517-6.

[11] G. E. Corazza and G. Ferrari, "New bounds for the Marcum Q-function", IEEE Trans. Inform. Theory, vol. 48, no. 11, pp. 3003-3008, Nov. 2002.

[12] M. Abramowitz and I. A. Stegun, Handbook of Mathematical Functions with Formulas, Graphs, and Mathematical Tables, NY, 9th ed., 1972.

[13] A. P. Prudnikov, Y. A. Brychkov, and O. I. Marichev, Integrals and Series. Vol. 3: More Special Functions, 2003.

[14] M. K. Simon and M.-S. Alouini, Digital Communication over Fading Channels: A Unified Approach to Performance Analysis, John Wiley \& Sons, Inc., 1st ed., 2000.

[15] M. K. Simon and M.-S. Alouini, "A unified approach to the performance analysis of digital communications over generalized fading channels", Proc. of the IEEE, vol. 86, no. 9, pp. 1860-1877, Sept. 1998.

[16] G. Ferrari and G. E. Corazza, "Tight bounds and accurate approximations for DQPSK transmission bit error rate", IEEE Electron. Lett., vol. 40, no. 20, pp. 1284-1285, Sept. 2004.

[17] G. H. Golub and J. H. Welsch, "Calculation of gauss quadrature rules", Math. Comput., vol. 23, no. 106, pp. 221-230, Apr. 1969. 